\title{
Henoch-Schönlein purpura following high-voltage electric burn injury: A case report and review of the literature
}

\author{
XUNHONG DUAN*, DONGQI YU*, CHANGLONG YU*, BIAO WANG and YIBIN GUO \\ Department of Burns and Plastic Surgery, The 175th Hospital of PLA, Affiliated Southeast Hospital of \\ Xiamen University, Zhangzhou, Fujian 363000, P.R. China
}

Received October 9, 2014; Accepted September 28, 2015

DOI: $10.3892 /$ etm.2015.2905

\begin{abstract}
Henoch-Schönlein Purpura (HSP) is a systemic vasculitis of unknown cause, with immune-mediated inflammation of the small vessels, which is characterized by a series of clinical symptoms, such as purpuric rash, colicky abdominal pain, arthritis and acute glomerulonephritis. Twenty-one days following a high-voltage electrical burn injury, a 40-year-old man developed classic clinical symptoms of HSP, including purpuric rash on bilateral lower extremities and abdominal pain. The patient was diagnosed with HSP associated with high-voltage burn injury, which is an extremely rare phenomenon. The diagnosis was based on the clinical manifestations of purpuric rash, abdominal pain and arthralgia, as well as the findings of laboratory examinations [increased levels of serum immunoglobulin $\mathrm{A}$ (11.6g/l) and complements C3 (9.6 g/l) and C4 (7.6 g/l), and a positive fecal occult blood test]. The patient was treated with antihistamines (loratadine tablets; $10 \mathrm{mg} /$ day), anti-inflammatory medication (methylprednisolone sodium succinate; $40 \mathrm{mg} /$ day) and oral omeprazole magnesium. The symptoms gradually decreased within 2 weeks from treatment and no abnormality was observed at the 3-, 6- and 12-month follow-ups. In patients who have suffered an electrical burn injury, this autoimmune disease may be caused by long-term inflammation. Therefore, examination of the liver and kidney functions of such patients is important in order to decrease the risk of post-traumatic immune system dysfunction.
\end{abstract}

Correspondence to: Professor Yibin Guo, Department of Burns and Plastic Surgery, The 175th Hospital of PLA, Affiliated Southeast Hospital of Xiamen University, 269 Zhanghua Middle Road, Zhangzhou, Fujian 363000, P.R. China

E-mail: gybsszxwk@163.com

\section{*Contributed equally}

Key words: abdominal pain, purpura, purpuric rash, electric burn injury

\section{Introduction}

Henoch-Schönlein Purpura (HSP) is a systemic leukocytoclastic vasculitis mediated by immunoglobulin A (IgA) (1) and characterized by a series of clinical features, including non-thrombocytopenic palpable purpura, abdominal pain, arthritis and kidney damage (2). At present, the exact causes of HSP is unclear. The condition most commonly associated with HSP is an upper respiratory infection; however, complications of surgical interventions, digestive perforation and massive gastrointestinal bleeding can also be due to HSP (3). HSP is known to be the most common form of vasculitis in children; more than $90 \%$ of HSP cases are $<10$ year-old pediatric patients (4). However, this condition also occurs in adults. The diagnosis of HSP is made on the basis of clinical manifestations, laboratory examinations and histopathological biopsy (5). The diagnostic criteria for this disease, however, vary from patient to patient. Treatment with antihistamines and anti-inflammatory and antispasmodic drugs can effectively relieve the symptoms. Since numerous organs can be involved in HSP, the outcome and prognosis depends on the lesions in those organs, particularly the kidney in pediatric patients (6). Adults who present with HSP are more likely to experience more severe complications, such as purpuric rash, abdominal pain, arthralgia, digestive perforation, massive gastrointestinal bleeding and nephritis; however, the induction of HSP following trauma is rarely reported in adults $(2,7)$. The present study reports the case of a 40 year-old man, who was diagnosed with HSP following a high-voltage electrical burn injury. Patient consent was obtained from the patient's family.

\section{Case report}

A 40 year-old man who suffered from a high-voltage burn injury affecting $2 \%$ of the total body surface area, with severe injury of the right hand (Fig. 1), was admitted to the Department of Burns and Plastic Surgery of the 175th Hospital of PLA, Affiliated Southeast Hospital of Xiamen University (Zhangzhou, China). Informed consent was obtained from the patient prior to the study. The patient underwent one-stage debridement surgery and was then moved to the intensive care unit and received antibiotic and intravenous fluid support. Following the first surgery, 


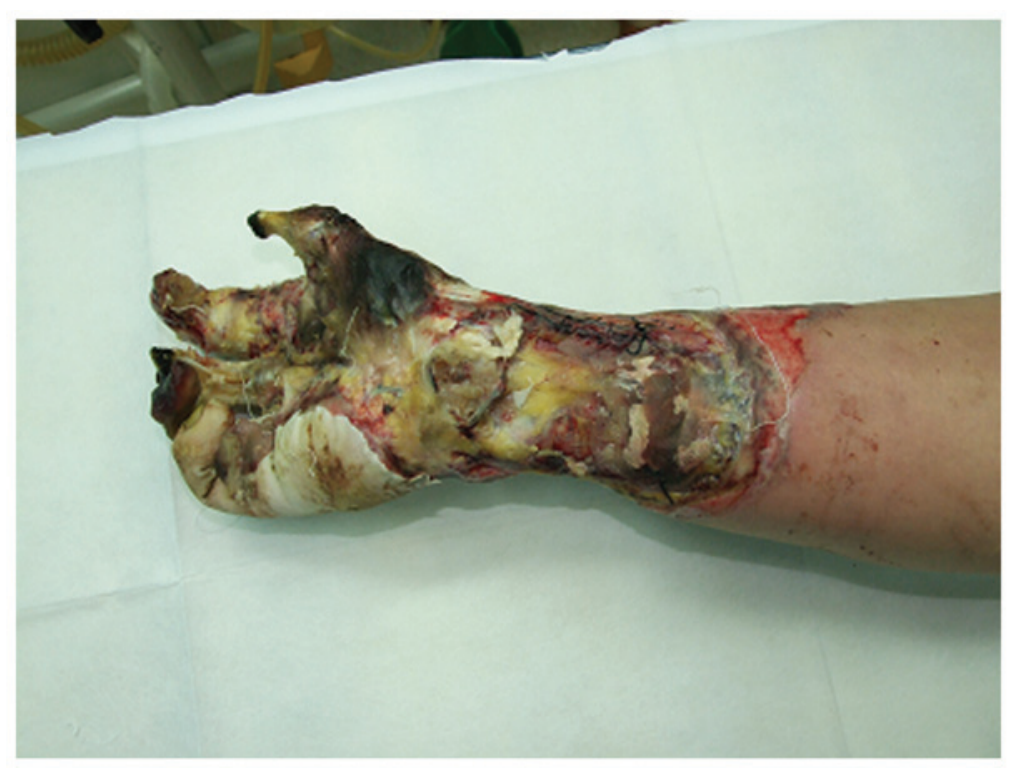

Figure 1. Electric burn injury of the right the hand of the patient.

the patient was subjected to three additional surgical procedures in the space of 2 weeks, and followed a routine postoperative course, which consisted of the administration of antibiotics and the frequent changing of dressings. In addition, the laboratory examinations showed no evident abnormalities. On week 3 following hospitalization, however, an interspersed erythema appeared on the patient's body. No other abnormalities were observed upon physical examination. Follow-up showed that the clinical symptoms, such as abdominal pain, arthralgia and purpuric rash (Fig. 2), improved gradually over the course of 3 days. On the basis of these symptoms, HSP was considered as a possible diagnosis and further examinations were performed. Prior to receiving the results of urine and stool routine tests (including a fecal occult blood test), the patient was treated with oral loratadine tablets (10 mg/day) and a single intramuscular injection of $5 \mathrm{mg}$ anisodamine. On the fifth day of the symptoms, the fecal occult blood test results came back positive, with a high volume of blood detected in the stool, which confirmed the diagnosis of HSP. The urine test results were normal. These results, in combination with increases in serum $\operatorname{IgA}(11.6 \mathrm{~g} / \mathrm{l})$ and complements C3 (9.6 g/l) and C4 (7.6 g/l) levels, led to the conclusion that the diagnosis of HSP was correct, despite the fact that the kidney function test was normal. With the injection of Solu-Medrol ${ }^{\circledR}$ (methylprednisolone sodium succinate; $40 \mathrm{mg} /$ day) and oral administration of omeprazole magnesium (40 mg/day), the abdominal pain was alleviated and the purpuric rash gradually decreased. Following a 2-week treatment, the symptoms were resolved, and on week 5 following admission, the patient had completely recovered and was discharged. At the 3-, 6- and 12-month follow-ups, the results of all laboratory tests, including kidney and liver function and urine and stool tests, were normal.

\section{Discussion}

HSP was first described in $1837(8,9)$ and is an autoimmune acute leucocytoclastic vasculitis, which most commonly involves the skin, joints, kidney and gastrointestinal system (10). HSP may

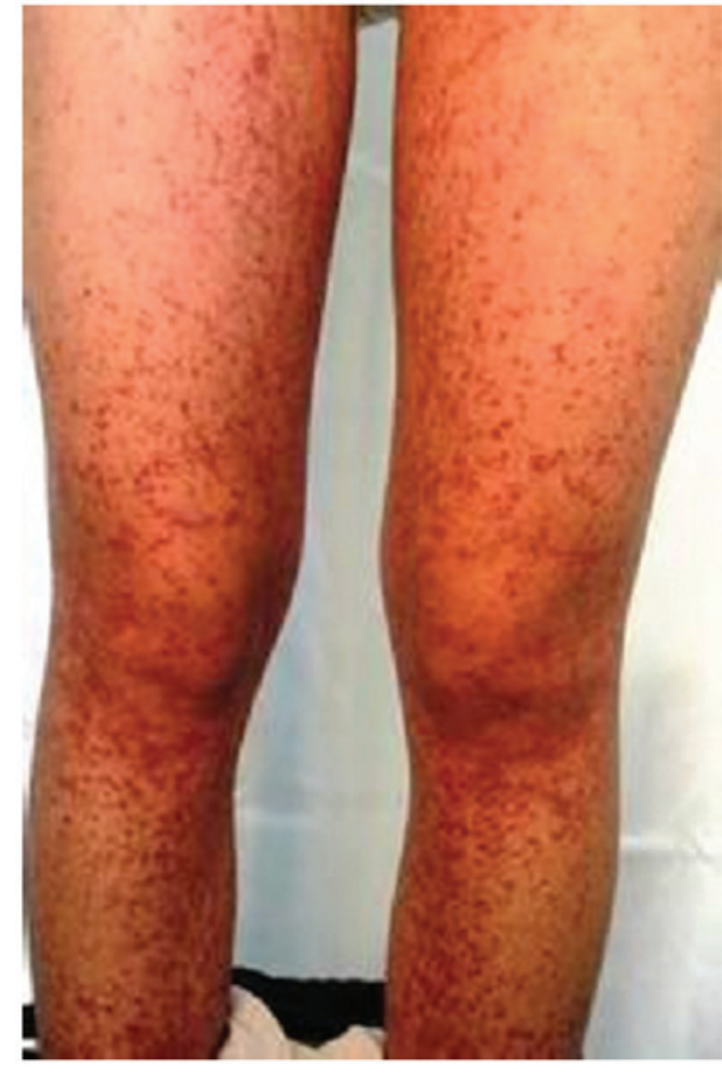

Figure 2. Rash on the bilateral lower extremities.

be related to streptococcal infections, viral infections, medicine taken, food sensitivity and insect bites (9), and it mainly affects $<10$ year-old pediatric patients (4). The prevalence is lower in adults, compared with pediatric patients; however, HSP should not be ignored in adults, particularly during hospitalization. The diagnostic criteria of HSP, described by the American College of Rheumatology (11) and the International Consensus Conference, $2006(12,5)$ are based on clinical manifestations, 
such as arthralgia/arthritis, diffuse abdominal pain, hematuria/proteinuria and purpura nephritis and pathohistological findings of leukocytoclastic vasculitis and IgA-immune deposits in vessel walls and/or glomeruli. Not every patient, however, presents with all aforementioned symptoms, which makes the establishment of standard criteria challenging. Briefly, it has been shown that cutaneous manifestations are presented in $70 \%$ of HSP cases (13), renal involvement in 20-60\% (14) and gastrointestinal symptoms in up to $85 \%$ (10). Timely urine routine and stool occult blood tests are required, particularly when the symptoms are not apparent, in order to avoid misdiagnosis or missed diagnosis. In the present case, all necessary laboratory examinations had been concluded within a day of admission, which helped prevent serious, even fatal, HSP complications, such as pulmonary hemorrhage or myocardial infarction (15). Effective therapy is of paramount importance. Analgesics or non-steroidal anti-inflammatory drugs are used as first-line treatment for the relief of arthralgia (10) and antihistamines for the treatment of purpuric rash. Glucocorticoids, such as methylprednisolone, are commonly used for the relief of abdominal and joint pain. Severe symptoms affecting the renal and central nervous systems may lead to life-threatening conditions, and immunosuppressive agents and plasmapheresis may be required (10); however, caution is required prior to the use of glucocorticoids and immunosuppressive agents, as subsequent infections may occur in cases where the patient has a wound caused by thermal or electric burn injuries.

The phenomenon of HSP following an electric burn injury is extremely rare as, to the best of our knowledge, only one other case has been reported (16). The association between electrical burn injury and HSP is not clearly established, but the present case of HSP following an electric burn injury was not considered by our institution as purely coincidental. Burn or electrical burn injuries can cause long-term inflammation through tissue necrosis and frequent changes in dressings, leading to the production of a considerable quantity of inflammatory mediators that potentially induce the appearance of HSP. This hypothesis, although a little far-fetched, provides the most likely explanation of the mechanism of this phenomenon, and requires further investigation.

In conclusion, HSP is a multisystem autoimmune disease, which most commonly involves the skin, joints, kidneys and gastrointestinal system. In patients with electric burn injury, this autoimmune disease may result from a long-term inflammation; therefore, examining the liver and kidney function of those patients is imperative, in order to decrease the risk of post-traumatic immune system dysfunction.

\section{References}

1. Cao N, Chen T, Guo ZP, Li MM and Jiao XY: Elevated serum levels of visfatin in patients with Henoch-Schönlein purpura. Ann Dermatol 26: 303-307, 2014.

2. Semeena N and Adlekha S: Henoch-Schönlein purpura associated with gangrenous appendicitis: A case report. Malays J Med Sci 21: 71-73, 2014.

3. Pan YX, Ye Q, Shang SQ, Mao JH, Zhang T, Shen HQ and Zhao N: Relationship between immune parameters and organ involvement in children with Henoch-Schonlein purpura. PLoS One 9: e115261, 2014.

4. Reamy BV, Williams PM and Lindsay TJ: Henoch-Schönlein Purpura. Am Fam Physician 80: 697-704, 2009.

5. Yang YH, Yu HH and Chiang BL: The diagnosis and classification of Henoch-Schönlein purpura: An updated review. Autoimmun Rev 13: 355-358, 2014.

6. Pillebout E and Verine J. Henoch-Schönlein purpura in the adult. Rev Med Interne 35: 372-381 (In French) 2014.

7. Tanaka M, Kitadai Y, Kodama M, Sumida T, Shinagawa K, Yoshioka K, Mitsuoka Y, Masuda H, Hiyama T, Tanaka S, et al: A case report of anaphylactoid purpura with acute abdominal pain secondary to trauma caused by traffic accident. Nihon Shokakibyo Gakkai Zasshi 105: 566-571, 2008 (In Japanese).

8. Schönlein JL (ed): Allegemeine und specielle Pathologie und Therapie. Vol 2. Literatur-Comptoir, Herisau, Switzerland, 1834 (In German).

9. Kraft DM, Mckee D and Scott C: Henoch-Schönlein purpura: A review. Am Fam Physician 58: 405-408, 1998.

10. Hasija N, Taxak S, Bhardwaj M and Vashist K: Anesthetic management of a patient with Henoch-Schonlein purpura for drainage of cervical lymphadenitis: A case report. Saudi J Anaesth 8: 282-283, 2014.

11. Mills JA, Michel BA, Bloch DA, Calabrese LH, Hunder GG, Arend WP, Edworthy SM, Fauci AS, Leavitt RY and Lie JT: The American College of Rheumatology 1990 criteria for the classification of Henoch-Schönlein purpura. Arthritis Rheum 33: 1114-1121, 1990.

12. Dillon MJ and Ozen S: A new international classification of childhood vasculitis. Pediatr Nephrol 21: 1219-1222, 2006.

13. Sinclair P: Henoch-schönlein purpura - a review. Curr Allergy Clin Immunol 23, 116-120, 2010.

14. Narchi H: Risk of long term renal impairment and duration of follow up recommended for Henoch-Schonlein purpura with normal or minimal urinary findings: A systematic review. Arch Dis Child 90: 916-920 2005.

15. Agraharkar M, Gokhale S, Le L, Rajaraman S and Campbell GA: Cardiopulmonary manifestations of Henoch-Schönlein purpura. Am J Kidney Dis 35: 319-322, 2000.

16. Zhang W, Xie WG, Min WX, Wang DY, Zhang J and Wang SY: Treatment of thoracic and abdominal cavity perforation complicated by Henoch-Schonlein purpura nephritis in a patient with high-voltage electric burn. Zhonghua Shao Shang Za Zhi 29: 454-458, 2013 (In Chinese). 